Global Mental Health

cambridge.org/gmh

\section{Other \\ Review}

All authors of this review equally contributed, but the author order was determined using a coin toss. Therefore, all authors should be read as the first author.

Cite this article: Hosen I, al-Mamun F, Mamun MA (2021). Prevalence and risk factors of the symptoms of depression, anxiety, and stress during the COVID-19 pandemic in Bangladesh: Mental Health 8, e47, 1-16. https://doi.org/

Received: 21 September 2021

Revised: 14 November 2021

Accepted: 29 November 2021

\section{Keywords:}

Anxiety; COVID-19 and psychological impact; depression; mental health in Bangladesh; prevalence and risk factors; PTSD; stress; systematic review

\section{Authors for correspondence:}

Ismail Hosen,

E-mail: ihosen1631@gmail.com;

Firoj al-Mamun,

E-mail: firojphiju@gmail.com;

Mohammed A. Mamun,

E-mail: mamunphi46@gmail.com

\title{
Prevalence and risk factors of the symptoms of depression, anxiety, and stress during the COVID-19 pandemic in Bangladesh: a systematic review and meta-analysis
} a systematic review and meta-analysis. Global 10.1017/gmh.2021.49

Ismail Hosen ${ }^{1,2}$ (D), Firoj al-Mamun ${ }^{1,2}$ (D) and Mohammed A. Mamun ${ }^{1,2}$ (D)

${ }^{1}$ CHINTA Research Bangladesh, Savar, Dhaka 1342, Bangladesh and ${ }^{2}$ Department of Public Health and Informatics, Jahangirnagar University, Savar, Dhaka 1342, Bangladesh

\section{Abstract}

The COVID-19 pandemic affects people's psychological well-being as well as their risk of physical complications. Under the circumstance, it is essential to synthesize the existing evidence on psychological consequences with a view to fostering policymaking. Thus, a systematic attempt was compiled to review the Bangladeshi literature related to common mental health problems (i.e. depression, anxiety, and stress) during the COVID-19 pandemic. Adhering to the PRISMA guidelines, a systematic literature search was performed using Medline or PubMed, Scopus, PsycINFO, Web of Science, CINAHL, Google Scholar, PsyArxiv, MedRxiv, and ResearchGate, between 20 December 2020 and 5 March 2021, followed by predetermined eligibility criteria. The inclusion criteria for this review were observational studies involving at least one mental health problem (i.e. stress, depression, and anxiety) published in peer-reviewed journals or preprint servers in the English language after the inception of the pandemic in Bangladesh. The pooled prevalence of depression, anxiety, and stress was $47 \%\left(95 \%\right.$ CI $\left.39-55 \%, I^{2}=99.14 \%\right), 47 \%\left(95 \%\right.$ CI $\left.39-54 \%, I^{2}=99.78 \%\right)$, and $44 \%\left(95 \%\right.$ CI $\left.30-58 \%, I^{2}=99.36 \%\right)$, respectively. Subgroup analysis revealed that students were experiencing a higher rate of depression, anxiety, and stress than general people and healthcare professionals. The associated risk factors of mental health problems were gender, age, residence area, family size, monthly family income, educational status, marital status, physical exercise, smoking, alcohol use, fear of COVID-19, presence of chronic illness, unemployment status, and exposure to COVID-19-related news and social media. This systematic review provides baseline data on the symptoms of depression, anxiety, and stress across various Bangladeshi cohorts, which are anticipated to be helpful to the respective authorities for implementing cohort-specific mental health strategies.

\section{Introduction}

The newly incepted Coronavirus Disease 2019 (COVID-19) pandemic has become the most challenging global issue. This contagious disease has already spread out within all countries and territories worldwide after its first appearance in late December 2019 at Wuhan, China, caused by a newly discovered virus named severe acute respiratory syndrome coronavirus 2 (SARS-CoV-2) (Puspitasari et al., 2020). Regardless of the unpredictable and uncontrolled situation of the viral infection, the World Health Organization declared this outbreak as a pandemic on 11 March 2020 (BBC News, 2020; Puspitasari et al., 2020). Consequently, approximately 577K cases had been infected with the virus on 23 March 2021, with 8720 deaths in Bangladesh.

People infected with the SARS-CoV-2 manifest both symptomatic and asymptomatic signs Cambridge University Press. This is an Open Access article, distributed under the terms of the Creative Commons AttributionNonCommercial-ShareAlike licence (http:// creativecommons.org/licenses/by-nc-sa/4.0), which permits non-commercial re-use, distribution, and reproduction in any medium, provided the same Creative Commons licence is used to distribute the re-used or adapted article and the original article is properly cited. The written permission of Cambridge University Press must be obtained prior to any commercial use.

\section{CAMBRIDGE UNIVERSITY PRESS} and symptoms, including fever, cough, fatigue, headache, myalgia, muscle pain, breath shortness, acute upper respiratory tract distress, diarrhea, etc. (CDC, 2020; Chen et al., 2020). Even though an individual is not infected with SARS-CoV-2, the higher rates of COVID-19-related infection and mortality have an impact on their quality of life, leading to a worsening psychological state. Moreover, to alleviate this contagious outbreak at the community level, some of the non-therapeutic public health interventions were implemented globally, including countrywide lockdown, home or institution-based isolation or quarantine, shutting down all kinds of public activities along with closing educational institutions, especially restriction in social and community movements, etc. (Dsouza et al., 2020; Gualano et al., 2020). Prior research found that people subjected to these measures experienced significant mental health consequences, which led to people disliking the importance of social restriction measures aimed at suppressing viral infection (Hawryluck et al., 2004).

In Bangladesh, the first COVID-19 case was identified on 8 March 2020 (Anwar et al., 2020). After some of that point time, the authority of Bangladesh seized countrywide 
lockdown with the title of 'general holidays' to mitigate the outbreak at the community level, which was a significant challenge for a lower- and middle-income country with limited resources in the healthcare settings (Anwar et al., 2020). Under these circumstances, many people were laid off or became unemployed, with no hope of finding new employment (Bhuiyan et al., 2020). Consequently, the jobless situation led people to suffer from higher rates of mental instabilities (Sultana et al., 2020). Due to mental problems related to unemployment and economic crisis, self-harm and suicidal incidences are also observed in Bangladesh. For instance, a study reported that all but one of the COVID-19-related suicide cases occurred as of economic and financial problems within the first 3 weeks of April 2020 (Bhuiyan et al., 2020). In addition, the uncertainty about going back to everyday life, fear of being infected, social and economic disruption, rising social negligence, difficulties in getting proper treatment as of the overwhelming number of patients, etc., generally increase psychological suffering, including post-traumatic stress disorder (PTSD), insomnia, phobia, fear, anxiety, depression, etc. (Hossain et al., 2020a; Sundarasen et al., 2020; World Health Organization, 2020).

In Bangladesh, after the first COVID-19 suicide incidence on 25 March 2020, epidemiological studies concerning mental health problems to identify risky individuals were recommended (Mamun and Griffiths, 2020), and many studies have already been conducted across the country. But there is no beyond a systematic evaluation to better understand the impact of the COVID-19 pandemic on people's psychological health. Therefore, this is the first systematic attempt to review Bangladeshi studies on COVID-19 pandemic-related mental health problems such as depression, anxiety, and stress. This systematic review and metaanalysis included those Bangladeshi studies reported mental health outcomes' prevalence and/or associated factors, and the findings reported herein are anticipated to be useful in facilitating appropriate mental health strategies.

\section{Methods}

\section{Search strategies}

For conducting a systematic review, the Preferred Reporting Items for Systematic Reviews and Meta-Analyses (PRISMA) guideline (Moher et al., 2010) adhered to the present review (PRISMA 2020 checklist is provided in the online Supplementary File). Literature searches were conducted between 20 December 2020 and 5 March 2021, using a group of bounded keywords in the relevant databases. The searching keywords included three types of items, (i) exposure (e.g. 'COVID-19', 'Coronavirus', SARS-Cov-2, 'COVID-19 pandemic'), (ii) outcome of interest (e.g. 'mental health', 'depression, anxiety and stress', 'psychological impact', 'depressive disorders', 'depression', 'anxiety disorder', 'anxiety', 'stress', 'stress disorders'), and (iii) country (e.g. 'Bangladesh'). The searches were conducted with the combination of these three types of keywords.

\section{Data eligibility criteria}

For being included in the present review, every article must fulfill the following requirement of (i) being a Bangladeshi study, (ii) being conducted after the inception of the COVID-19 pandemic, (iii) being an observational study (cross-sectional or longitudinal) in nature, (iv) being concerned with at least one common mental health problem (i.e. PTSD or stress, depression, and anxiety), (v) reporting the prevalence and/or risk factors of these disorders, (vi) being published in peer-reviewed journals or preprint servers, (vii) being published in the English language.

\section{Data collection procedure}

First of all, a comprehensive literature search was conducted using the databases of Medline or PubMed to identify articles related to the prevalence and/or associated factors of depression, anxiety and stress reported during the COVID-19 pandemic in Bangladesh. Later on, Scopus, PsycINFO, Web of Science, CINAHL, Google Scholar, and ResearchGate were used to retrieve missing peer-reviewed articles. Additionally, preprint servers such as PsyArxiv and MedRxiv were also searched to find relevant articles. Therefore, after a preliminary search conducted by the principal investigator, other investigators subsequently re-circled the procedure to ensure articles were not excluded from this review. As a result, a total of 175 articles were identified, whereas the 'titles and abstracts' of the retrieved articles were scanned to adhere to the study inclusion criteria of the present review. To the end, a total of 24 articles were included in this review after confirming all of the requirements (Fig. 1).

\section{Data extraction}

In Microsoft Excel, a data extraction file was designed to make necessary changes from the recruited articles. After that, data were demonstrated by following records: information about the author and publication year, study design, sampling and data collection method, specific group and sample size, time for data collection, assessments tool along with cutoff value and the prevalence and risk factors of PTSD, stress, depression, and anxiety by the principal investigator. Finally, after the independent investigation and cross-checking by all the investigators, the disagreements were solved in the final file, as presented in Table 1.

\section{Quality assessment of the included studies}

The risk of bias of the included studies was assessed by The Joanna Briggs Institute (JBI) checklist used for prevalence studies. The JBI uses a four-point Likert scale with answers being 'no', 'yes', 'unclear', or 'not applicable', for the following questions: (1) appropriateness of the sample frame (Was the sample frame appropriate to address the target population?); (2) recruitment procedure (Were study participants sampled in an appropriate way?); (3) adequacy of the sample size (Was the sample size adequate?); (4) description of subjects and setting (Were the study subjects and the setting described in detail?); (5) description of the identified sample (Was the data analysis conducted with sufficient coverage of the identified sample?); (6) validity of the methods used to screen for mental health problems (Were valid methods used for the identification of the mental health problems?); (7) reliability of the methods used to screen for mental health problems (Were the mental health problems measured in a standard, reliable way for all participants?); (8) adequacy of statistical analyses (Was there appropriate statistical analysis?); and (9) response rate (Was the response rate adequate, and if not, was the low response rate managed appropriately?). Articles were assigned one point per yes. Details of the quality assessment criteria can be found in the online Supplementary Materials. 


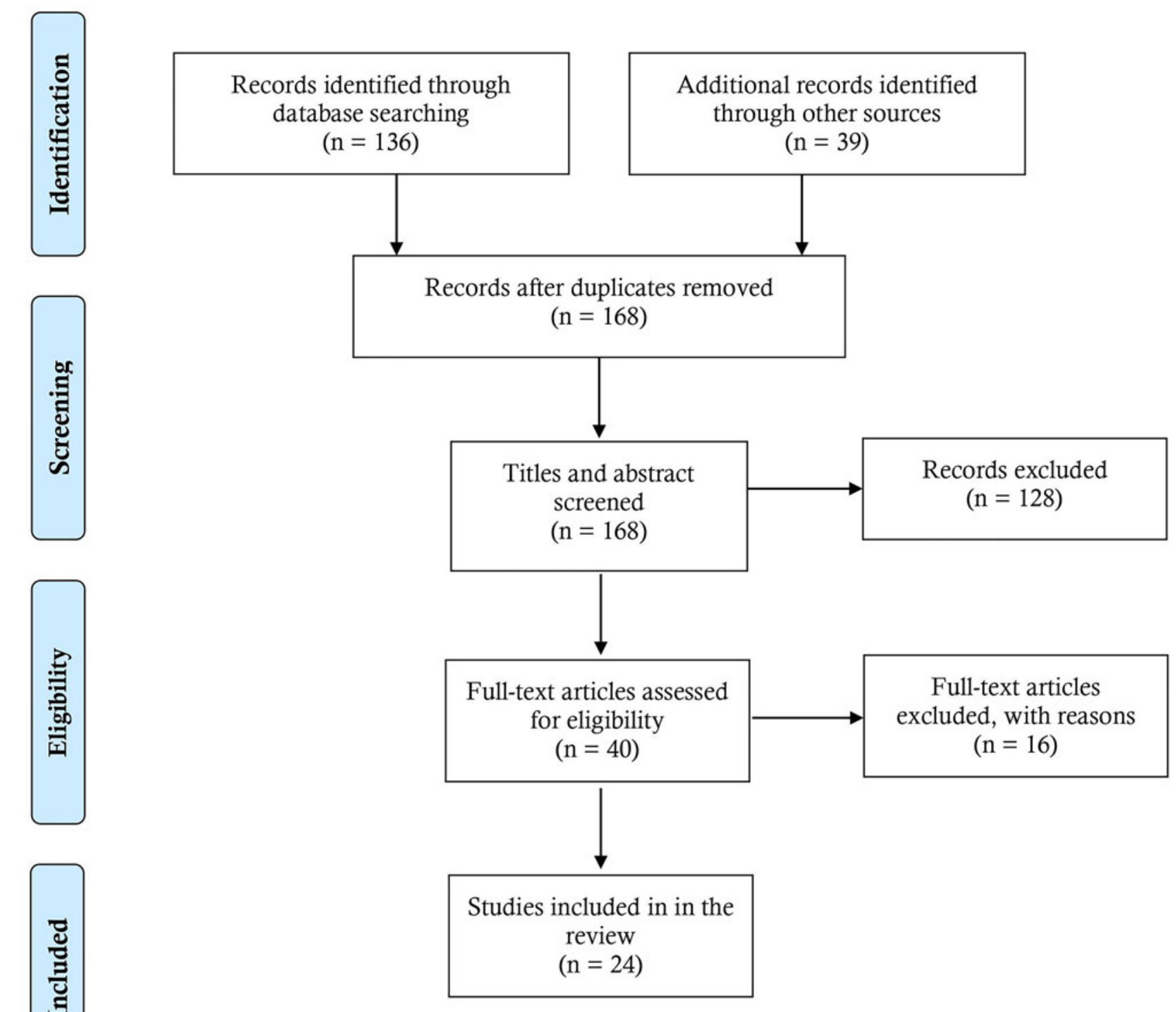

Fig. 1. PRISMA flow diagram.

\section{Statistical analysis}

Random-effect models were used in the study, assuming significant heterogeneity in all of the studies. The heterogeneity estimated of the effect of the sample size was calculated as $I^{2}$ statistic. $I^{2}$ values of $<25,25-75$, and $75 \%$ or more indicated low, moderate, and substantial heterogeneity, respectively. Cochran's $Q$ test and $\tau^{2}$ statistics were used to assess the heterogeneity between studies. Subgroup analysis was performed based on the specific population group in the study. The results are presented in Forest plots as an estimate with a 95\% confidence interval. The publication was assessed using the funnel plots to show the biasness graphically. In addition, Egger's test was applied for rigorous assessment of publication bias. Subgroup analysis was presented if there were three or more studies available for analysis. All the analysis was conducted using the STATA software version 16.

\section{Results}

\section{General description of the included studies}

A total of 24 studies were encompassed in the present review, whereas a total of 49806 respondents participated autonomously, including a range of sample size 114-15 543; whereas the mean age of the participants ranged between $21.4( \pm 2)$ and 31.41 ( \pm 8.73$)$ years. All of the included studies were cross-sectional and were conducted through online media and platforms. Most studies were surveyed among general population $(n=9)$ including different cohorts, e.g. students $(n=7)$, healthcare professionals $(n=4)$, quarantined individuals $(n=1)$, wage-earners $(n=1)$, SARS-CoV-2 infected individuals $(n=1)$, and bankers $(n=1)$. In addition, utmost participants belonged to younger adult age groups. Details of the included study description can be found in Table 1. 
Table 1. Characteristics of the included literature in the present review

\begin{tabular}{|c|c|c|c|c|c|c|c|c|}
\hline $\begin{array}{l}\text { Authors and } \\
\text { publication } \\
\text { year }\end{array}$ & $\begin{array}{l}\text { Study design and } \\
\text { sampling } \\
\text { procedure }\end{array}$ & $\begin{array}{l}\text { Data } \\
\text { collection } \\
\text { method and } \\
\text { time }\end{array}$ & $\begin{array}{l}\text { Sample } \\
\text { size }\end{array}$ & $\begin{array}{l}\text { Specific group and } \\
\text { mean age }\end{array}$ & $\begin{array}{l}\text { Assessment scale } \\
\text { with cutoff score }\end{array}$ & $\begin{array}{l}\text { Assessment } \\
\text { criteria }\end{array}$ & $\begin{array}{l}\text { Prevalence } \\
\text { rate(s) }\end{array}$ & Risk factors \\
\hline $\begin{array}{l}\text { Sayeed et al. } \\
(2020)\end{array}$ & $\begin{array}{l}\text { Cross-sectional; } \\
\text { snowball }\end{array}$ & $\begin{array}{l}\text { Web survey; } \\
29 \text { April-7 } \\
\text { May } 2020\end{array}$ & 589 & Students, age: NR & $\begin{array}{l}\text { DASS- } 21 ; \text { cutoff: } \\
\text { anxiety } \geqslant 7 \\
\text { depression } \geqslant 10 \\
\text { stress } \geqslant 11\end{array}$ & $\begin{array}{l}\text { Mild to } \\
\text { extremely } \\
\text { severe }\end{array}$ & $\begin{array}{l}\text { A: } 26.6 \% \\
\text { D: } 61.9 \% \\
\text { S: } 57.05 \%\end{array}$ & $\begin{array}{l}\text { A: Gender, age, education, family income, } \\
\text { residence, family size, negative COVID-19 } \\
\text { perceptions, and COVID-19-related } \\
\text { symptoms and social stressors } \\
\text { D: Gender, age, education, family income, } \\
\text { residence, family size, under-5 children in } \\
\text { the family, and negative COVID-19 } \\
\text { perceptions } \\
\text { S: Gender, age, under-5 children in the } \\
\text { family, negative COVID-19 perceptions, and } \\
\text { COVID-19-related symptoms and social } \\
\text { stressors }\end{array}$ \\
\hline $\begin{array}{l}\text { Al Banna } \\
\text { et al. }(2020)\end{array}$ & $\begin{array}{l}\text { Cross-sectional; } \\
\text { convenience and } \\
\text { snowball }\end{array}$ & $\begin{array}{l}\text { Online } \\
\text { survey; } 29 \\
\text { April-7 May } \\
2020\end{array}$ & 1427 & $\begin{array}{l}\text { General population; } \\
\text { age: } 25.75 \pm 6.75 \\
\text { years }\end{array}$ & $\begin{array}{l}\text { DASS-21; cutoff: } \\
\text { anxiety }>6, \\
\text { depression }>9 \\
\text { stress }>10\end{array}$ & $\begin{array}{l}\text { Mild to } \\
\text { extremely } \\
\text { severe }\end{array}$ & $\begin{array}{l}\text { A: } 33.7 \% \\
\text { D: } 57.9 \% \\
\text { S: } 59.7 \%\end{array}$ & $\begin{array}{l}\text { A: Gender, age, marital status, occupation, } \\
\text { education, residence, family size, elderly } \\
>50 \text { years in family and negative COVID- } 19 \\
\text { perceptions } \\
\text { D: Gender, age, marital status, education, } \\
\text { elderly }>50 \text { years in family, and negative } \\
\text { COVID-19 perceptions } \\
\text { S: Gender, marital status, occupation, } \\
\text { education, family income, and negative } \\
\text { COVID-19 perceptions }\end{array}$ \\
\hline $\begin{array}{l}\text { Rahman } \\
\text { et al. (2020) }\end{array}$ & $\begin{array}{l}\text { Cross-sectional; } \\
\text { convenience and } \\
\text { snowball }\end{array}$ & $\begin{array}{l}\text { Online } \\
\text { survey; } 6 \\
\text { June-27 } \\
\text { June } 2020\end{array}$ & 614 & $\begin{array}{l}\text { General population; } \\
\text { NR }\end{array}$ & $\begin{array}{l}\text { DASS-21; cutoff: } \\
\text { NR }\end{array}$ & $\begin{array}{l}\text { A and D: } \\
\text { Moderate to } \\
\text { extremely } \\
\text { severe } \\
\text { S: Mild to } \\
\text { moderate }\end{array}$ & $\begin{array}{l}\text { A: } 37 \% \\
\text { D: } 38.23 \% \\
\text { S: } 25.38 \%\end{array}$ & $\begin{array}{l}\text { D: Gender, age, marital status, occupation, } \\
\text { education, physical health conditions, } \\
\text { contact with COVID-19 cases, } \\
\text { COVID-19-related psychological and } \\
\text { socio-economic conditions } \\
\text { A: Gender, age, marital status, current } \\
\text { location, occupation, education, physical } \\
\text { health conditions, contact with COVID-19 } \\
\text { cases, COVID-19-related psychological and } \\
\text { socio-economic conditions } \\
\text { S: Gender, age, occupation, education, } \\
\text { physical health conditions, contact with } \\
\text { covID-19 cases, CovID-19-related } \\
\text { psychological and socio-economic } \\
\text { conditions }\end{array}$ \\
\hline $\begin{array}{l}\text { Islam et al. } \\
\text { (2020c) }\end{array}$ & $\begin{array}{l}\text { Cross-sectional; } \\
\text { NR }\end{array}$ & $\begin{array}{l}\text { Online } \\
\text { survey; } 5 \\
\text { May-15 May } \\
2020\end{array}$ & 340 & $\begin{array}{l}\text { Adult population; } \\
\text { age: } 26.23 \pm 6.39 \\
\text { years }\end{array}$ & $\begin{array}{l}\text { Self-developed } \\
\text { (single item, yes/ } \\
\text { no) }\end{array}$ & $\begin{array}{l}\text { Binary } \\
\text { category }\end{array}$ & S: $85.6 \%$ & $\begin{array}{l}\text { S: Gender, age, marital status, and } \\
\text { occupation, fear of self and/or family } \\
\text { member, and/or relative COVID-19 infection, } \\
\text { hampering study and career plan, and } \\
\text { financial difficulties }\end{array}$ \\
\hline $\begin{array}{l}\text { Barua et al. } \\
(2020)\end{array}$ & $\begin{array}{l}\text { Cross-sectional; } \\
\text { convenience }\end{array}$ & $\begin{array}{l}\text { Online } \\
\text { survey; } 1 \\
\text { April-30 May } \\
2020\end{array}$ & 370 & $\begin{array}{l}\text { Frontline doctors; } \\
\text { age: } 30.5 \pm 4.4 \text { years }\end{array}$ & $\begin{array}{l}\text { PHQ-4 (GAD 2+ } \\
\text { PHQ 2); cutoff: } \\
\text { both depression } \\
\text { and anxiety } \geqslant 3\end{array}$ & Mild to severe & $\begin{array}{l}\text { A: } 36.5 \% \\
\text { D: } 38.4 \%\end{array}$ & $\begin{array}{l}\text { A and/or D: Gender, shifting duty, } \\
\text { inadequate resources, sleep disturbance, } \\
\text { presence of chronic disease, number of } \\
\text { chronic conditions, asthma and } \\
\text { hypertension }\end{array}$ \\
\hline
\end{tabular}




\begin{tabular}{|c|c|c|c|c|c|c|c|c|}
\hline $\begin{array}{l}\text { Sultana et al. } \\
(2020)\end{array}$ & $\begin{array}{l}\text { Cross-sectional; } \\
\text { convenience } \\
\text { sampling }\end{array}$ & $\begin{array}{l}\text { Online } \\
\text { survey; } 20 \\
\text { May-30 May } \\
2020\end{array}$ & 707 & $\begin{array}{l}\text { Wage earners; age: } \\
31.41 \pm 8.73 \text { years }\end{array}$ & $\begin{array}{l}\text { PHQ-9 and GAD-7; } \\
\text { cutoff: anxiety } \\
\geqslant 10 \text {, depression } \\
\geqslant 10\end{array}$ & $\begin{array}{l}\text { Moderate to } \\
\text { severe }\end{array}$ & $\begin{array}{l}\text { A: } 58.6 \% \\
\text { D: } 55.9 \%\end{array}$ & $\begin{array}{l}\text { Both A and D: Gender, education, } \\
\text { occupation, family income, and current } \\
\text { financial situation (e.g. not getting any } \\
\text { salary, no earning source, salary not } \\
\text { enough for family, not satisfied with } \\
\text { earning, upcoming financial crisis, } \\
\text { children's education loss, increased price of } \\
\text { daily necessary commodities, inadequate } \\
\text { food supply, etc.) }\end{array}$ \\
\hline $\begin{array}{l}\text { Ripon et al. } \\
(2020)\end{array}$ & $\begin{array}{l}\text { Cross-sectional; } \\
\text { NR }\end{array}$ & $\begin{array}{l}\text { Online } \\
\text { survey; till } \\
19 \text { October } \\
2020\end{array}$ & 5792 & $\begin{array}{l}\text { Adult quarantine } \\
\text { population; age: NR }\end{array}$ & $\begin{array}{l}\text { CES-D and IES-R; } \\
\text { cutoff: depression } \\
\geqslant 16, \text { PTSD } \geqslant 20\end{array}$ & $\begin{array}{l}\text { Standard } \\
\text { cutoff points }\end{array}$ & $\begin{array}{l}\text { D: } 24 \% \\
\text { PTSD: } 35 \%\end{array}$ & $\begin{array}{l}\text { PTSD: Socio-demographic risk factors are } \\
\text { not reported. Nutritional behavior changes, } \\
\text { the likelihood of infection, nutritional } \\
\text { perceived efficiency, exaggeration of risk, } \\
\text { eating or taking substances, the severity of } \\
\text { the fear. }\end{array}$ \\
\hline $\begin{array}{l}\text { Hasan et al. } \\
(2020)\end{array}$ & $\begin{array}{l}\text { Cross-sectional; } \\
\text { convenience } \\
\text { sampling }\end{array}$ & $\begin{array}{l}\text { Online } \\
\text { survey; } 21 \\
\text { April-10 May } \\
2020\end{array}$ & 412 & Physicians; NR & $\begin{array}{l}\text { HADS; Cutoff: } \\
\text { higher score } \\
\text { indicates higher } \\
\text { level of symptoms }\end{array}$ & NR & $\begin{array}{l}\text { A: } 67.72 \% \\
\text { D: } 48.5 \%\end{array}$ & $\begin{array}{l}\text { A and/or D: Gender, experienced symptoms } \\
\text { of COVID-19, had not received incentives/ } \\
\text { just commitments, self-funded source of } \\
\text { PPE, inadequate training, lacking perceived } \\
\text { self-efficacy to control COVID-19-positive } \\
\text { patients, severe tension of getting infected } \\
\text { with COVID-19, extreme tension about } \\
\text { family members getting infected with } \\
\text { COVID-19, check daily news/update more } \\
\text { than or equal to } 4 \text { times, difficulty to stay } \\
\text { away from media, hard to stay away from } \\
\text { social media, daily leisure time }<2 \mathrm{~h} \text {, didn't } \\
\text { earn enough to support the family during } \\
\text { the pandemic, afraid of getting assaulted } \\
\text { on the way to home or hospital, sleep } \\
\text { disturbances, being agitated more usually }\end{array}$ \\
\hline $\begin{array}{l}\text { Tasnim et al. } \\
(2020)\end{array}$ & $\begin{array}{l}\text { Cross-sectional; } \\
\text { NR }\end{array}$ & $\begin{array}{l}\text { Online } \\
\text { survey; } \\
\text { between } \\
\text { July and } \\
\text { August } 2020\end{array}$ & 803 & $\begin{array}{l}\text { Healthcare } \\
\text { professionals; age: } \\
27.3 \pm 6.9 \text { years }\end{array}$ & $\begin{array}{l}\text { HADS (both } \\
\text { depression and } \\
\text { anxiety } \geqslant 8 \text { ) }\end{array}$ & $\begin{array}{l}\text { HADS; cutoff: } \\
\text { both } \\
\text { depression } \\
\text { and } \\
\text { anxiety } \geq 8\end{array}$ & $\begin{array}{l}\text { A: } 69.5 \% \\
\text { D: } 39.5 \%\end{array}$ & $\begin{array}{l}\text { A and D: Gender, having moderate and poor } \\
\text { health status, irregular physical exercise, } \\
\text { smoking, having regrets about their } \\
\text { profession because of the pandemic and } \\
\text { many unexpected experiences, experiencing } \\
\text { discrimination in the workplace, and facing } \\
\text { social problems due to working in a lab or } \\
\text { hospital }\end{array}$ \\
\hline $\begin{array}{l}\text { Ahmed et al. } \\
(2020)\end{array}$ & $\begin{array}{l}\text { Cross-sectional; } \\
\text { NR }\end{array}$ & $\begin{array}{l}\text { Online } \\
\text { survey; } 2020\end{array}$ & 500 & $\begin{array}{l}\text { General population; } \\
\text { age: } 25.95 \pm 7.57 \\
\text { years }\end{array}$ & $\begin{array}{l}\text { DASS-21; cutoff: } \\
\text { anxiety } \geqslant 4 \text {, } \\
\text { depression } \geqslant 5 \text {, } \\
\text { stress } \geqslant 8\end{array}$ & $\begin{array}{l}\text { Mild to } \\
\text { extremely } \\
\text { severe }\end{array}$ & $\begin{array}{l}\text { A: } 39.8 \% \\
\text { D: } 43 \% \\
\text { S: } 34.8 \%\end{array}$ & $\begin{array}{l}\text { D, A, and S: Gender, educational level, } \\
\text { occupation, marital status, and age }\end{array}$ \\
\hline $\begin{array}{l}\text { Hossain } \\
\text { et al. }(2020 c)\end{array}$ & $\begin{array}{l}\text { Cross-sectional; } \\
\text { NR }\end{array}$ & $\begin{array}{l}\text { Online } \\
\text { survey; 3rd } \\
\text { week of } \\
\text { March } 2020\end{array}$ & 880 & $\begin{array}{l}\text { General population; } \\
\text { age: } 26.3 \pm 7.2 \text { years }\end{array}$ & $\begin{array}{l}\text { GAD-7; cutoff: } \\
\text { anxiety } \geqslant 10\end{array}$ & NR & A: $49.1 \%$ & $\begin{array}{l}\text { A: Social media exposure over } 4 \mathrm{~h} \text { a day, } \\
\text { bad health condition and self-rated health }\end{array}$ \\
\hline $\begin{array}{l}\text { Islam et al. } \\
(2020 d)\end{array}$ & $\begin{array}{l}\text { Cross-sectional; } \\
\text { snowball }\end{array}$ & $\begin{array}{l}\text { Online } \\
\text { survey; } 6 \\
\text { May-12 May } \\
2020\end{array}$ & 476 & $\begin{array}{l}\text { University students; } \\
\text { age: NR }\end{array}$ & $\begin{array}{l}\text { GAD-7 and PHQ-9; } \\
\text { cutoff: anxiety } \geqslant 5 \text {, } \\
\text { depression } \geqslant 5\end{array}$ & $\begin{array}{l}\text { Mild to } \\
\text { extremely } \\
\text { severe }\end{array}$ & $\begin{array}{l}\text { A: } 81.8 \% \\
\text { D: } 82.4 \%\end{array}$ & $\begin{array}{l}\mathrm{D} \text { and } \mathrm{A} \text { : Private tuition during COVID-19, } \\
\text { lagging academically, living with family }\end{array}$ \\
\hline
\end{tabular}


Table 1. (Continued.)

\begin{tabular}{|c|c|c|c|c|c|c|c|c|}
\hline $\begin{array}{l}\text { Authors and } \\
\text { publication } \\
\text { year }\end{array}$ & $\begin{array}{l}\text { Study design and } \\
\text { sampling } \\
\text { procedure }\end{array}$ & $\begin{array}{l}\text { Data } \\
\text { collection } \\
\text { method and } \\
\text { time }\end{array}$ & $\begin{array}{l}\text { Sample } \\
\text { size }\end{array}$ & $\begin{array}{l}\text { Specific group and } \\
\text { mean age }\end{array}$ & $\begin{array}{l}\text { Assessment scale } \\
\text { with cutoff score }\end{array}$ & $\begin{array}{l}\text { Assessment } \\
\text { criteria }\end{array}$ & $\begin{array}{l}\text { Prevalence } \\
\text { rate(s) }\end{array}$ & Risk factors \\
\hline $\begin{array}{l}\text { Islam et al. } \\
(2020 a)\end{array}$ & $\begin{array}{l}\text { Cross-sectional; } \\
\text { NR }\end{array}$ & $\begin{array}{l}\text { Online } \\
\text { survey; } 29 \\
\text { March-6 } \\
\text { April } 2020\end{array}$ & 1311 & $\begin{array}{l}\text { General population; } \\
\text { Age: } 23.54 \pm 4.97 \\
\text { years }\end{array}$ & $\begin{array}{l}\text { GAD-7; cutoff: } \\
\text { anxiety } \geqslant 10\end{array}$ & $\begin{array}{l}\text { Moderate to } \\
\text { extremely } \\
\text { severe }\end{array}$ & A: $37.3 \%$ & $\begin{array}{l}\text { A: Gender, age, education level, married, } \\
\text { occupation, panic }\end{array}$ \\
\hline $\begin{array}{l}\text { Mamun et al. } \\
\text { (2020) }\end{array}$ & $\begin{array}{l}\text { Cross-sectional; } \\
\text { NR }\end{array}$ & $\begin{array}{l}\text { Online } \\
\text { survey; } 1 \\
\text { April-10 } \\
\text { April } 2020\end{array}$ & 10067 & $\begin{array}{l}\text { General population; } \\
\text { age: } 29.9 \pm 9.6 \text { years }\end{array}$ & $\begin{array}{l}\text { PHQ-9; cutoff: } \\
\text { depression } \geqslant 10\end{array}$ & NR & D:33.3\% & $\begin{array}{l}\text { D: Gender, age, smoking, having comorbid } \\
\text { diseases and insomnia, having high scores } \\
\text { on the Fear COVID-19 Scale }\end{array}$ \\
\hline $\begin{array}{l}\text { Al Zubayer } \\
\text { et al. }(2020)\end{array}$ & $\begin{array}{l}\text { Cross-sectional; } \\
\text { NR }\end{array}$ & $\begin{array}{l}\text { Online } \\
\text { survey; } 1 \\
\text { June-10 } \\
\text { June } 2020\end{array}$ & 1146 & $\begin{array}{l}\text { General population; } \\
\text { age: } 26.38 \pm 8.87 \\
\text { years }\end{array}$ & $\begin{array}{l}\text { DASS- } 21 ; \text { cutoff: } \\
\text { anxiety } \geqslant 10 \\
\text { depression } \geqslant 14 \\
\text { stress } \geqslant 19\end{array}$ & $\begin{array}{l}\text { Moderate to } \\
\text { extremely } \\
\text { severe }\end{array}$ & $\begin{array}{l}\text { A: } 46 \% \\
\text { D: } 47.2 \% \\
\text { S: } 32.5 \%\end{array}$ & $\begin{array}{l}\text { D, A, and S: Following COVID-19-related } \\
\text { news daily, having COVID-19 symptoms, } \\
\text { having direct and indirect contact with } \\
\text { COVID-19-infected people, fear of COVID-19 } \\
\text { infection }\end{array}$ \\
\hline $\begin{array}{l}\text { Safa et al. } \\
(2021)\end{array}$ & $\begin{array}{l}\text { Cross-sectional; } \\
\text { convenience and } \\
\text { snowball }\end{array}$ & $\begin{array}{l}\text { Online } \\
\text { survey; } 21 \\
\text { April-10 May } \\
2020\end{array}$ & 425 & $\begin{array}{l}\text { Medical students; } \\
\text { age: } 22 \pm 1.81 \text { years }\end{array}$ & $\begin{array}{l}\text { HADS; cutoff: both } \\
\text { depression and } \\
\text { anxiety }>8\end{array}$ & Mild to severe & $\begin{array}{l}\text { A: } 65.9 \% \\
\text { D: } 49.9 \%\end{array}$ & $\begin{array}{l}\text { A: Fear of getting infected with COVID-19, } \\
\text { fear of getting assaulted or humiliated on } \\
\text { the way to hospital or home, lack of } \\
\text { concentration on the study, agitation } \\
\text { D: Gender, fear of getting infected with } \\
\text { COVID-19, agitation }\end{array}$ \\
\hline $\begin{array}{l}\text { Khan et al. } \\
(2020)\end{array}$ & $\begin{array}{l}\text { Cross-sectional; } \\
\text { convenience } \\
\text { sampling }\end{array}$ & $\begin{array}{l}\text { Online } \\
\text { survey; } 9-23 \\
\text { April } 2020\end{array}$ & 505 & $\begin{array}{l}\text { College and } \\
\text { university students; } \\
\text { age: NR }\end{array}$ & $\begin{array}{l}\text { DASS-21; cutoff: } \\
\text { NR }\end{array}$ & $\begin{array}{l}\text { Mild to } \\
\text { extremely } \\
\text { severe }\end{array}$ & $\begin{array}{l}\text { A: } 33.3 \% \\
\text { D: } 46.92 \% \\
\text { S: } 28.5 \%\end{array}$ & $\begin{array}{l}\text { A: Cough, fatigue, fever, throat pain, } \\
\text { difficulty breathing, financial uncertainty, } \\
\text { fear of infection, inadequate food supply, } \\
\text { exposure to COVID-19 news in social media } \\
\text { and mass media } \\
\text { D: Fatigue, difficulty breathing, recreational } \\
\text { activity (e.g. watching TV series, reading } \\
\text { storybooks, online and offline gaming, etc.), } \\
\text { physical exercise, household chores, } \\
\text { financial uncertainty, inadequate food } \\
\text { supply, exposure to COVID-19 news in social } \\
\text { and mass media } \\
\text { S: Perceived COVID-19 symptoms (i.e. dry } \\
\text { cough, fever, difficulty breathing), } \\
\text { recreational activity (e.g. watching TV } \\
\text { series, reading storybooks, online and } \\
\text { offline gaming, etc.), financial uncertainty, } \\
\text { exposure to COVID-19 news in social media } \\
\text { and mass media }\end{array}$ \\
\hline $\begin{array}{l}\text { Faisal et al. } \\
(2021)\end{array}$ & $\begin{array}{l}\text { Cross-sectional; } \\
\text { snowball }\end{array}$ & $\begin{array}{l}\text { Online } \\
\text { survey; } 10 \\
\text { April to } 24 \\
\text { April } 2020\end{array}$ & 874 & $\begin{array}{l}\text { University students; } \\
\text { age: } 22.83 \pm 2.79 \\
\text { years }\end{array}$ & $\begin{array}{l}\text { GAD-7 and } \\
\text { CESD-R-10; cutoff: } \\
\text { anxiety } \geqslant 10, \\
\text { depression } \geqslant 10\end{array}$ & $\begin{array}{l}\text { A: Moderate to } \\
\text { severe } \\
\text { D: NR }\end{array}$ & $\begin{array}{l}\text { A: } 40 \% \\
\text { D: } 72 \%\end{array}$ & $\begin{array}{l}\text { A and D: Worrying about the effects of } \\
\text { COVID-19 }\end{array}$ \\
\hline
\end{tabular}




\begin{tabular}{|c|c|c|c|c|c|c|c|c|}
\hline $\begin{array}{l}\text { Islam et al. } \\
(2020 b)\end{array}$ & $\begin{array}{l}\text { Cross-sectional; } \\
\text { convenience } \\
\text { sampling }\end{array}$ & $\begin{array}{l}\text { Online } \\
\text { survey; } \\
\text { during April } \\
2020\end{array}$ & 3122 & $\begin{array}{l}\text { University students; } \\
\text { age: } 21.4 \pm 2 \text { years }\end{array}$ & $\begin{array}{l}\text { DASS-21; cutoff: } \\
\text { anxiety } \geqslant 8 \\
\text { depression } \geqslant 10 \\
\text { stress } \geqslant 15\end{array}$ & $\begin{array}{l}\text { At least mild } \\
\text { symptoms }\end{array}$ & $\begin{array}{l}\text { A: } 71.5 \% \\
\text { D: } 76.1 \% \\
\text { S: } 70.1 \%\end{array}$ & $\begin{array}{l}\text { Both } D, A \text {, and } S: \text { Gender, being older ( } 25- \\
29 \text { years), having } \geqslant 5 \text { family members, living } \\
\text { in urban areas, not engaging in physical } \\
\text { exercise, having dissatisfaction with their } \\
\text { sleep, spending more hours browsing the } \\
\text { Internet, having dissatisfaction with } \\
\text { academic studies under the present } \\
\text { COVID-19 circumstances, and smoking }\end{array}$ \\
\hline $\begin{array}{l}\text { Sakib et al. } \\
(2021)\end{array}$ & $\begin{array}{l}\text { Cross-sectional; } \\
\text { NR }\end{array}$ & $\begin{array}{l}\text { Online } \\
\text { survey; } \\
\text { between } 8 \\
\text { April and } 25 \\
\text { April } 2020\end{array}$ & 3388 & $\begin{array}{l}\text { General population; } \\
\text { age: } 30.11 \pm 6.44 \\
\text { years }\end{array}$ & $\begin{array}{l}\text { PHQ-9; cutoff: } \\
\text { depression } \geqslant 10\end{array}$ & NR & D: $27.8 \%$ & $\begin{array}{l}\text { Gender, marital status, and having chronic } \\
\text { physical diseases, having children }\end{array}$ \\
\hline $\begin{array}{l}\text { Mina et al. } \\
\text { (2021) }\end{array}$ & $\begin{array}{l}\text { Cross-sectional; } \\
\text { NR }\end{array}$ & $\begin{array}{l}\text { Online } \\
\text { survey; } 26 \\
\text { May-27 June } \\
2020\end{array}$ & 153 & $\begin{array}{l}\text { COVID-19-infected } \\
\text { people; age: } 39.43 \pm \\
17.59 \text { years }\end{array}$ & $\begin{array}{l}\text { GAD-7 and PHQ-9; } \\
\text { cutoff: both } \\
\text { anxiety and } \\
\text { depression } \geqslant 10\end{array}$ & $\begin{array}{l}\text { Moderate to } \\
\text { extremely } \\
\text { severe }\end{array}$ & $\begin{array}{l}\text { A: } 63.5 \% \\
\text { D: } 56.6 \%\end{array}$ & NR \\
\hline $\begin{array}{l}\text { Dhar et al. } \\
(2020)\end{array}$ & $\begin{array}{l}\text { Cross-sectional; } \\
\text { NR }\end{array}$ & NR & 15543 & $\begin{array}{l}\text { University students; } \\
\text { age: NR }\end{array}$ & GAD-7; cutoff: NR & NR & $\begin{array}{l}\text { A: Mild } 3.82 \% \text {, } \\
\text { moderate } \\
48.41 \% \text {, } \\
\text { severe } \\
44.59 \%\end{array}$ & $\begin{array}{l}\text { A: Gender, living in the urban area, living } \\
\text { with their parents, financial condition, } \\
\text { infection of COVID-19 among relatives or } \\
\text { friends }\end{array}$ \\
\hline $\begin{array}{l}\text { Yasmin et al. } \\
\text { (2021) }\end{array}$ & $\begin{array}{l}\text { Cross-sectional; } \\
\text { purposive }\end{array}$ & $\begin{array}{l}\text { Online } \\
\text { survey; } 17 \\
\text { June to } 25 \\
\text { June } 2020\end{array}$ & 248 & Bankers; age: NR & $\begin{array}{l}\text { DASS-21; cutoff: } \\
\text { anxiety, } \\
\text { depression, stress } \\
\geqslant 21\end{array}$ & $\begin{array}{l}\text { Severely to } \\
\text { extremely }\end{array}$ & $\begin{array}{l}\text { A: } 10.6 \% \\
\text { D: } 12.1 \% \\
\text { S: } 11.1 \%\end{array}$ & $\begin{array}{l}\text { S: Gender, relationship status, having an } \\
\text { older adult and a pregnant woman at } \\
\text { home, having fair knowledge on COVID-19, } \\
\text { colleagues infected by COVID-19, smoking } \\
\text { more frequently, having a bad dream } \\
\text { during sleep } \\
\text { A: Living with relatives, colleagues infected } \\
\text { by COVID-19, using public transportation, } \\
\text { having a bad dream during sleep, } \\
\text { misbehaving with family members and } \\
\text { beaten children } \\
\text { D: Living with relatives, having an elderly } \\
\text { person and a pregnant woman at home, } \\
\text { fear of getting infected, smoking more } \\
\text { frequently, having a bad dream during } \\
\text { sleep }\end{array}$ \\
\hline $\begin{array}{l}\text { Khatun et al. } \\
(2021)\end{array}$ & $\begin{array}{l}\text { Cross-sectional; } \\
\text { NR }\end{array}$ & $\begin{array}{l}\text { Online } \\
\text { survey; } 4 \\
\text { May-10 May } \\
2020\end{array}$ & 114 & Physicians; age: NR & $\begin{array}{l}\text { GAD-7 and PHQ-9; } \\
\text { cutoff: anxiety } \geqslant 9 \\
\text { depression } \geqslant 10\end{array}$ & NR & $\begin{array}{l}\text { A: } 32.5 \% \\
\text { D: } 34.2 \%\end{array}$ & $\begin{array}{l}\text { A: Work per day ( } 8 \mathrm{~h} \text { or more) and current } \\
\text { job location (Dhaka division) } \\
\text { D: Gender, age, and marital status }\end{array}$ \\
\hline
\end{tabular}

A, anxiety; D, depression; S, stress; PTSD, post-traumatic stress disorders; NR, not reported; HADS, Hospital Anxiety and Depression Scale; DASS-21, Depression, Anxiety, Stress Scale 21; IES-R, Impact of Event Scale-Revised; CES-D, Center for Epidemiologic Studies-Depression; MDD, major depressive disorder; CESD-R-10, Center for Epidemiologic Studies Depression Scale-Revised. 


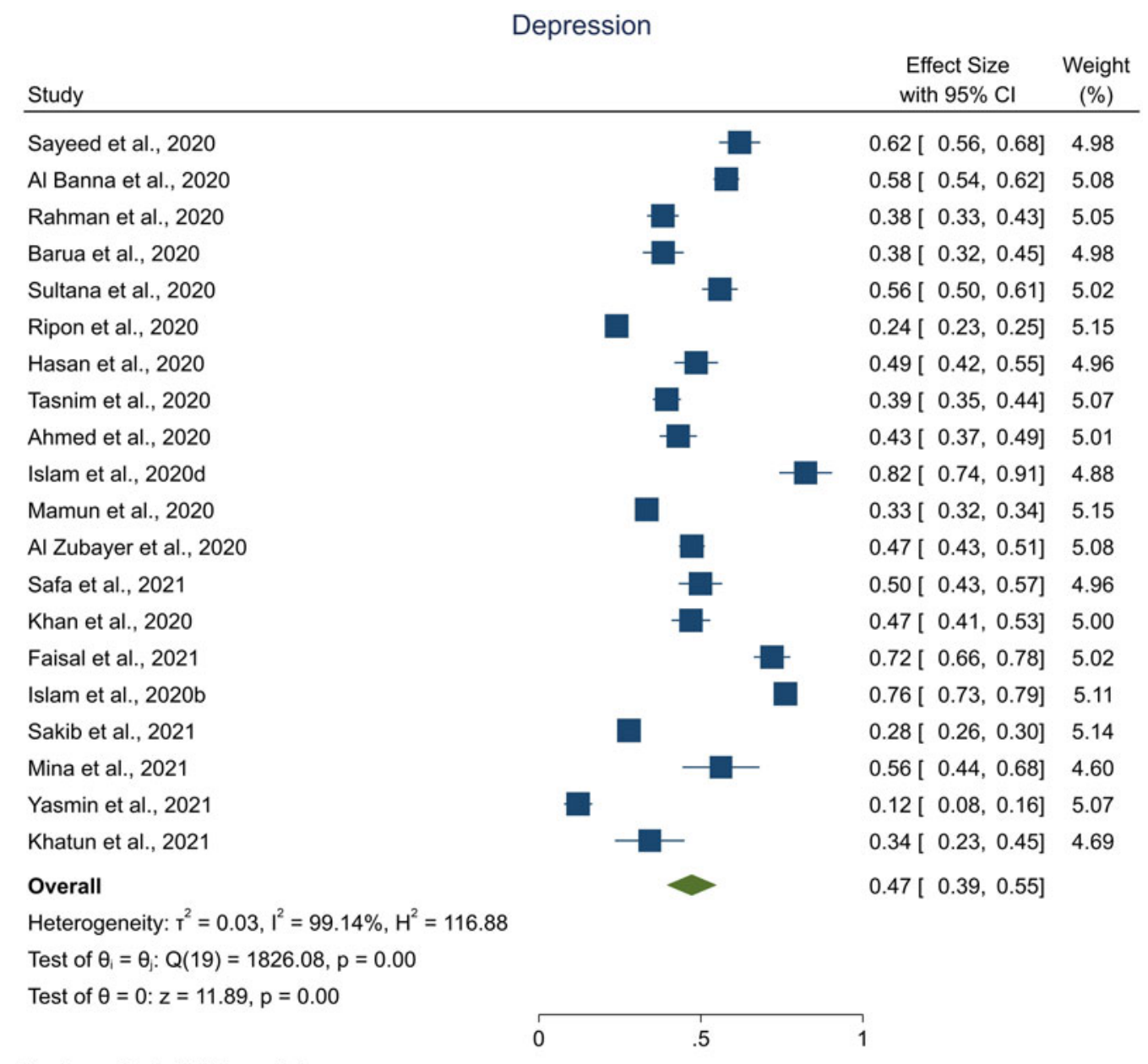

Fig. 2. Pooled prevalence of depression.

\section{Measurements used in the included studies}

Different asessment tools were used to detect mental health problems, whereas most studies utilized the DASS-21 $(n=7)$. There are also disparities in the prevalence cutoff values; that is, some of the studies $(n=8)$ considered 'mild to severe or extremely severe conditions' for identifying prevalence rates, whereas 'moderate to severe or extremely severe' in six studies, and nine studies did not report (or self-considered) the cutoff points. The description of the recruited articles' measurement use can be found in Table 1.

\section{Overall prevalence of depression, anxiety, and stress}

Prevalence of depression was reported in a total of 20 studies, the pooled estimated prevalence was $47 \%$ (95\% CI $39-55 \%$, $I^{2}=99.14 \%$ ) (Fig. 2). Higher rates of depression were reported among the university student's cohort $(82.4 \%)$, and lower rates were reported for bankers (i.e. 12.1\%). Where the prevalence of anxiety was reported among 20 studies, the pooled estimated prevalence was $47 \%$ (95\% CI 39-54\%, $I^{2}=99.78 \%$ ) (Fig. 3). Higher rates of anxiety (81.8\%) were observed among university students, whereas the rate was lower for bankers (10.6\%). A total of ten studies reported the prevalence of stress, the pooled estimated prevalence was $44 \%$ (95\% CI 30-58\%, $I^{2}=99.36 \%$ ) (Fig. 4). General people reported a higher level of stress $(85.6 \%)$, whereas lower was reported among the bankers (11.1\%).

\section{Group-specific prevalence of depression, anxiety, and stress}

Subgroup analysis was graphically presented using Forest plots. Please refer to the online Supplementary Materials. Here, subgroup analysis was presented if the number of available studies was 3 or more.

Prevalence of depression, anxiety, and stress: general population

Subgroup analysis of the general people against depression, anxiety, and stress was shown in Table 2. The results showed that the overall pooled prevalence of depression was $41 \%$ (95\% CI $\left.33-50 \%, I^{2}=98.46 \%\right)$. For anxiety, the overall estimated pooled prevalence was $40 \% \quad\left(95 \%\right.$ CI $\left.36-45 \%, \quad I^{2}=88.10 \%\right)$. Furthermore, for stress, the overall prevalence was 38\% (95\% CI $\left.23-53 \%, I^{2}=98.11 \%\right)$. 


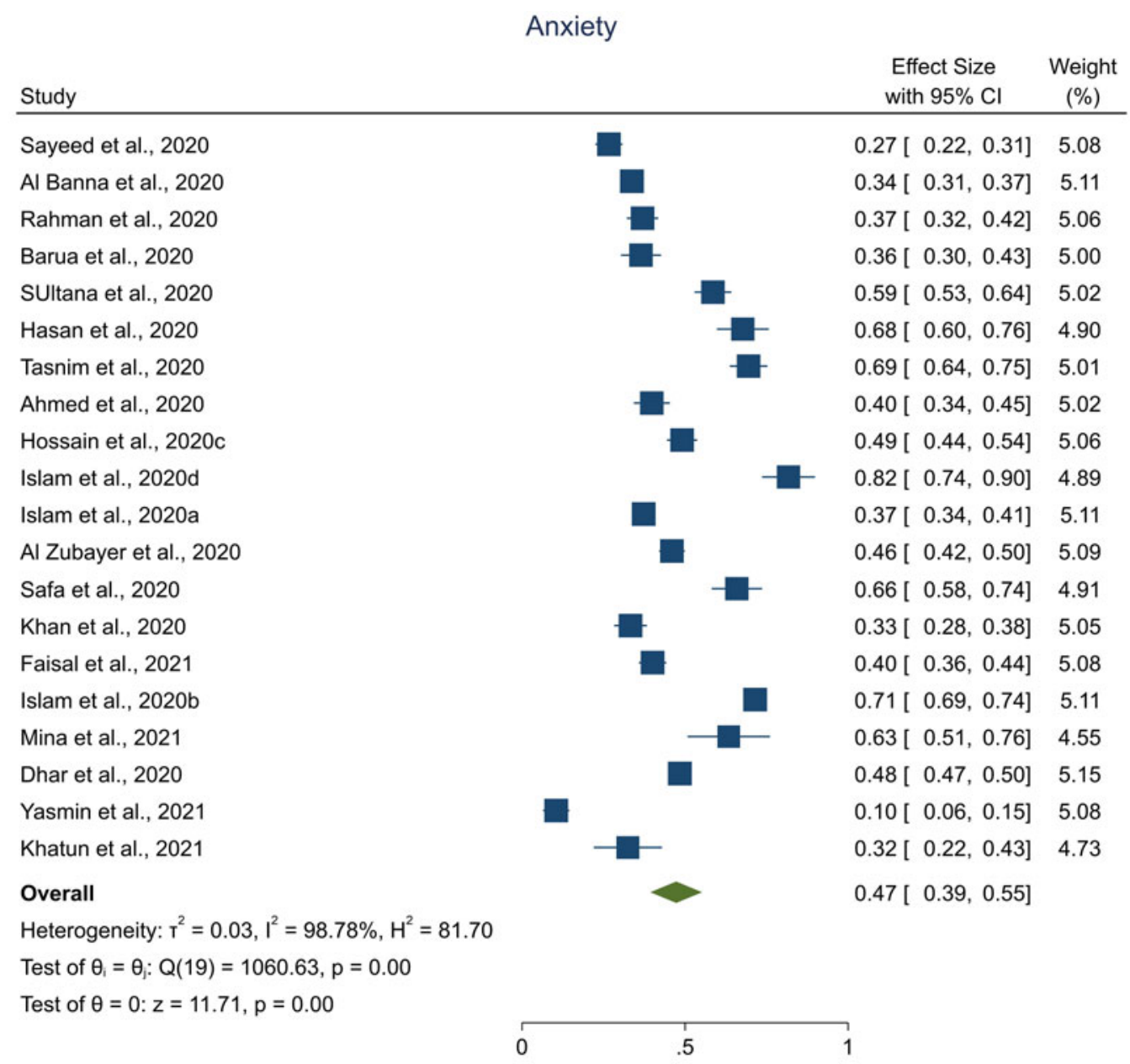

Random-effects REML model

Fig. 3. Pooled prevalence of anxiety.

\section{Stress}

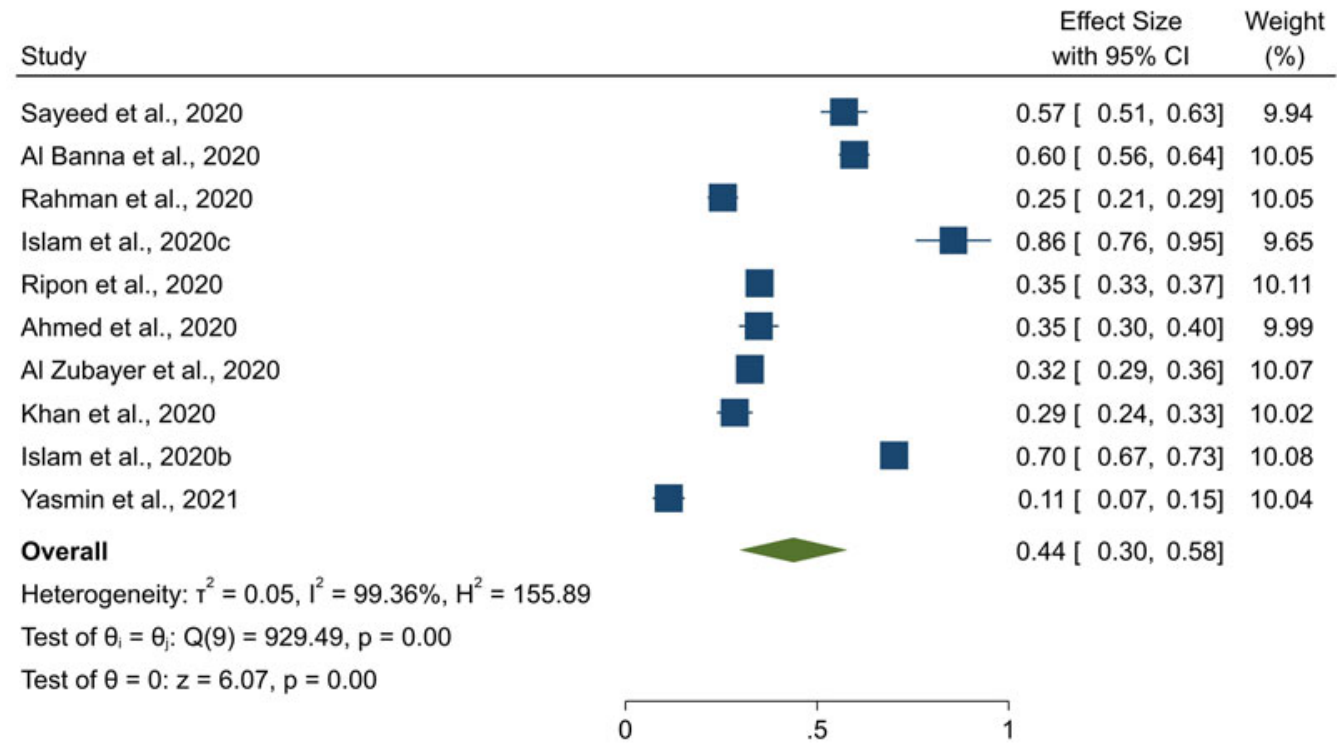

Fig. 4. Pooled prevalence of stress. 


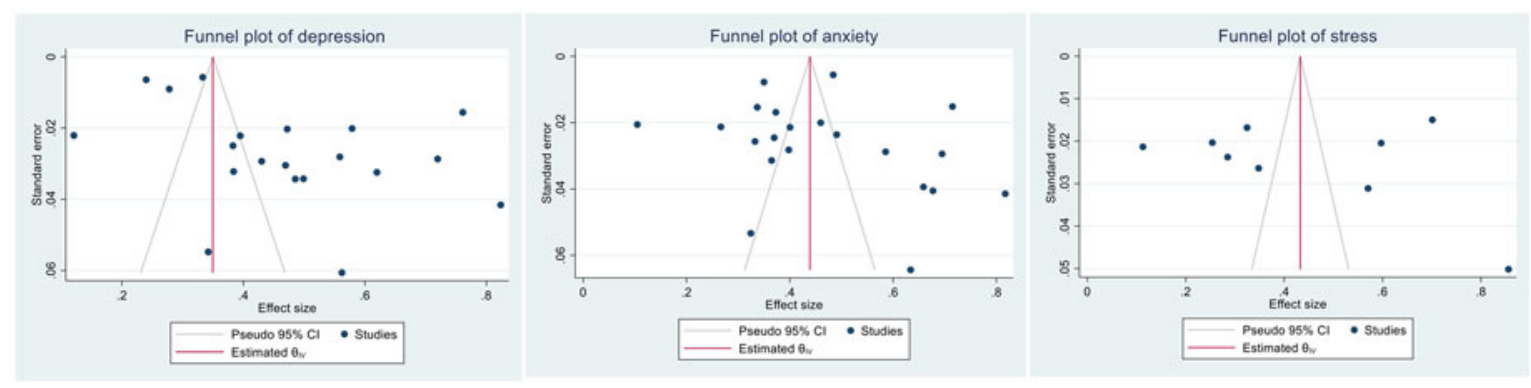

Fig. 5. Funnel plots for depression, anxiety, and stress.

Table 2. Subgroup analysis of specific group in terms of depression, anxiety, and stress

\begin{tabular}{lccc}
\hline Specific group & Depression & Anxiety & Stress \\
\hline General people & $41 \%\left(95 \% \mathrm{Cl} 33-50 \%, I^{2}=98.46 \%\right)$ & $40 \%\left(95 \% \mathrm{Cl} 36-45 \%, I^{2}=88.10 \%\right)$ & $38 \%\left(95 \% \mathrm{Cl} 23-53 \%, I^{2}=98.11 \%\right)$ \\
\hline Healthcare professionals & $41 \%\left(95 \% \mathrm{Cl} 35-46 \%, I^{2}=62.10 \%\right)$ & $52 \%\left(95 \% \mathrm{Cl} 32-71 \%, I^{2}=96.51 \%\right)$ & - \\
\hline Students & $65 \%\left(95 \% \mathrm{Cl} 53-76 \%, I^{2}=96.11 \%\right)$ & $52 \%\left(95 \% \mathrm{Cl} 37-68 \%, I^{2}=99.72 \%\right)$ & $52 \%\left(95 \% \mathrm{Cl} 28-76 \%, I^{2}=98.88 \%\right)$ \\
\hline
\end{tabular}

Prevalence of depression, anxiety, and stress: healthcare professionals

Subgroup analysis by a specific group in terms of depression, anxiety, and stress was presented in Table 2. The overall prevalence of depression was $41 \%\left(95 \%\right.$ CI $\left.35-46 \%, I^{2}=62.10 \%\right)$. Whereas it was $52 \%\left(95 \%\right.$ CI $\left.32-71 \%, I^{2}=96.51 \%\right)$ for anxiety.

\section{Prevalence of depression, anxiety, and stress: students}

Table 2 visualized the subgroup analysis of students in terms of depression, anxiety, and stress. Overall estimated prevalence of depression was $65 \%\left(95 \%\right.$ CI $\left.53-76 \%, I^{2}=96.11 \%\right)$. Similarly, for anxiety and stress, the pooled prevalence was $52 \%$ (95\% CI $\left.37-68 \%, I^{2}=99.72 \%\right)$ and $52 \%\left(95 \%\right.$ CI $\left.28-76 \%, I^{2}=98.88 \%\right)$, respectively.

Prevalence of depression, anxiety, and stress: bankers

During the COVID-19 pandemic, banks were not closed down, which might influence the bankers attaining higher mental health problems as they had to go to banks through public transportation mostly. Therefore, fear of being infected also increases their risk of being affected mentally. Nevertheless, only a study was directed among bankers regarding the level of anxiety, depression, and stress, which includes 10.6, 12.1, and $11.1 \%$, respectively; the lowest prevalence rates as identified across all of the cohorts included in this review (Yasmin et al., 2021).

\section{Prevalence of depression, anxiety, and stress: quarantined people}

Quarantine or restriction of movement had been introduced to switch the transmission of SARS-CoV-2 during the COVID-19 pandemic, wherever strategies associated with quarantine were constantly washing hands, wearing masks, and departing to the hospital when experiencing any complications related to COVID-19 symptoms (Ripon et al., 2020). Nevertheless, these effective measures had been reported to psychologically impact the quarantined people by evolving personalized trauma, fear, and uncertainty of their natural life, etc. However, following the situation, only a study was conducted among the quarantined people, whereas the quarantined people reported experiencing $24 \%$ and $35 \%$ depression and PTSD, respectively (Ripon et al., 2020).

Prevalence of depression, anxiety, and stress: wage-earners As of the COVID-19-related lockdown (e.g. movement restriction and earning losses), the wage-earning people became unable to bear the basic family needs, which effect on them being psychologically more vulnerable. Thus, as reported in a study, higher mental health problems were reported to this cohort; that is, $58.6 \%$ and $55.9 \%$ were the prevalence of anxiety and depression (Sultana et al., 2020).

\section{Prevalence of depression, anxiety, and stress: COVID-19-infected people}

People infected with the COVID-19 generally experience a higher level of psychological problems, including depression and anxiety as a fear of mortality. Higher mental health problems were observed in this group as a vulnerable cohort, e.g. $63.5 \%$ and $56.6 \%$ for anxiety and depression. Hence, only a study was directed among this vulnerable cohort (Mina et al., 2021).

\section{Risk factors associated with depression, anxiety, and stress}

The risk factors for common mental health problems can be divided into three major categories, that is, (i) socio-demographic, (ii) behavior and health, and (iii) COVID-19-related risk factors. The basic socio-demographic risk factors included age, gender, family income, marital status, education, residence and family type, and having elderly and children in the family. In contrast, the behavior and health-related risk factors were smoking and alcohol use, social media exposure, physical exercise, physical health conditions, and psychological conditions. Finally, COVID-19-related risk factors included insufficient protective resources, COVID-19-related perceptions, fear of COVID-19 infection, experiencing COVID-19-related symptoms, being suspected/infected with the COVID-19, being contacted with the COVID-19 cases, being quarantined or isolated, etc. 


\section{Socio-demographic risk factors}

Age

Age had been found significant in several studies that influenced mental health consequences (Ahmed et al., 2020; Al Banna et al., 2020; Islam et al., 2020a, 2020b, 2020c; Mamun et al., 2020; Rahman et al., 2020; Sayeed et al., 2020; Sultana et al., 2020; Khatun et al., 2021). Whereas Sayeed et al. (2020) found participants' age $\leq 22$ years were at $4.49,4.46$, and threefold higher risk of developing depression, anxiety, and stress respectively than age more than 22 years. However, another study reported that being aged between 24 and 39 than $\geqslant 40$ years had a lower risk of being anxious, but those aged $\leqslant 23$ years compared to $\geqslant 40$ years had experienced higher depressive symptoms (Al Banna et al., 2020). Similarly, Mamun et al. (2020) reported that younger people develop depressive symptoms mostly. Besides, Islam et al. (2020a) found that participants aged $13-20$ and $21-30$ years were at a lower risk of anxiety than more than 30 years. Another study reported that participants' being younger were at greater risk of developing psychological sufferings (Islam et al., 2020b). Furthermore, respondents aged below 25 years were approximately 2.29 times more prone to depression suffering than those more than 35 years old (Sultana et al., 2020). The included studies to this review randomly utilized different age ranges; hence, concluding age-based mental health risk factors can be limited.

\section{Gender}

Gender was found to be a significant predictor of mental health problems in a large number of studies (Ahmed et al., 2020; Al Banna et al., 2020; Barua et al., 2020; Dhar et al., 2020; Hasan et al., 2020; Islam et al., 2020a, 2020b, 2020c; Mamun et al., 2020; Rahman et al., 2020; Sayeed et al., 2020; Sultana et al., 2020; Tasnim et al., 2020; Khatun et al., 2021; Safa et al., 2021; Sakib et al., 2021; Yasmin et al., 2021). For example, a study found that females were suffering mostly from depression, anxiety, and stress, respectively, where it was 3.4, 3.4, and 4.5 times higher than males (Sayeed et al., 2020). Similarly, Al Banna et al. (2020) reported that male participants were reported significantly less vulnerable to stress, anxiety, and depression. Consistent with this, other studies also confirmed that female participants were at greater risk of those sufferings (Ahmed et al., 2020; Islam et al., 2020a; Mamun et al., 2020; Tasnim et al., 2020; Safa et al., 2021). However, no studies considered transgender concerning mental health in Bangladesh; although Mamun et al. (2020) collected data on it, transgender was excluded from the analyses concerning the presentation of mental health problems.

\section{Family monthly income}

Only two studies found family income as a significant factor in psychological sufferings (Sayeed et al., 2020; Sultana et al., 2020). Participants' monthly family income $\leqslant 27000$ BDT [Bangladeshi Taka (currency)] was at approximately 2.56- and 2.62-folds higher risk of developing anxiety and depressive symptoms, respectively, than $\geqslant 27000$ BDT (Sayeed et al., 2020). However, another study concerning the wage-earning participants reported that the average monthly family income to be associated with both symptoms of anxiety and depression; that is, 3.31 and 4.12 times higher risk, respectively, were observed for less than 30000 BDT compared to more than 70000 BDT (Sultana et al., 2020).

\section{Marital status}

Marital status had been found significant in a total of six studies (Ahmed et al., 2020; Islam et al., 2020a, 2020c; Khatun et al., 2021; Sakib et al., 2021; Yasmin et al., 2021), which depicted that a married person was at approximately two times higher risk of suffering from anxiety than an unmarried person (Islam et al., 2020a). But heterogeneity of depression rate distribution with marital status was also found in a comparative study among the general population and healthcare professionals; that is, the divorced participants reported the highest depression rate followed by those who were unmarried and married (53.5, 33.6, and $23.7 \%$, respectively) (Sakib et al., 2021). Similarly, physicians are either unmarried, divorced, widowed, or separated compared to married ones experiencing three times higher depressive symptoms (Khatun et al., 2021).

\section{Education}

Education has been a significant factor for depression, anxiety, and stress in some of the studies (Ahmed et al., 2020; Al Banna et al., 2020; Islam et al., 2020a; Rahman et al., 2020; Sayeed et al., 2020; Sultana et al., 2020; Yeasmin et al., 2020). A study claimed that higher secondary and undergraduate education participants were at less risk of stress than the graduate (Al Banna et al., 2020). Contradictorily, respondents with secondary and undergraduate education were approximately $12.87-$ and 1.12 -fold times higher risk of anxiety symptoms than the graduates (Al Banna et al., 2020). Additionally, the secondary education cohort was at a 2.30 times higher risk of suffering from depression than the graduates (Al Banna et al., 2020). Another study reported that participants with higher secondary education were more prone to develop depression and anxiety (Ahmed et al., 2020). Anxiety symptoms were less in having intermediate (11th-12th grade) education (about 0.5 times) and having bachelor education (approximately 0.64 times) than those with having higher education (above bachelor) (Islam et al., 2020a).

\section{Residence and family size}

Living areas and the size of families were independently found as the risk factors for mental health suffering among individuals (Dhar et al., 2020; Islam et al., 2020b; Sayeed et al., 2020; Yeasmin et al., 2020). For example, living in an urban area and families with more than five members reported having significant psychological issues (Dhar et al., 2020; Islam et al., 2020b; Yeasmin et al., 2020). In contrast, another study reported that having children less than five in a family experienced more mental health outcomes (Sayeed et al., 2020).

\section{Having elderly and children in the family}

Having elderly and children in the family increase the risk of mental health problems (Al Banna et al., 2020; Sayeed et al., 2020; Sakib et al., 2021). For instance, student participants having children $<5$ years in the family had experienced higher levels of depression and stress (Sayeed et al., 2020). Similarly, other studies reported that elderly $>50$ years old in the participants' families who were at an increased risk of COVID-19 experienced higher anxiety and depression symptoms (Al Banna et al., 2020; Sakib et al., 2021). 


\section{Behavior and health-related risk factors}

Smoking and alcohol use

Smoking and alcohol use significantly influenced the risk of mental health problems such as stress, depression, and anxiety in a few studies (Islam et al., 2020b; Mamun et al., 2020; Tasnim et al., 2020; Yasmin et al., 2021). For example, Mamun et al. (2020) reported that $15.6 \%$ of the participants were depressed who were involved with smoking, whereas this rate was 3.3\% for alcohol users. Besides, another study reported that those who were smoking more frequently significantly experienced a high score for stress and depression (Yasmin et al., 2021).

\section{Social media exposure}

Propaganda, falsehood, conspiracy theories, etc., related to the pandemic were increased, whereas social media was found to be one of the prime sources for collecting COVID-19-related information (Hasan et al., 2020). Therefore, constantly using social media was a significant risk factor for mental health sufferings, as some studies found (Hasan et al., 2020; Hossain et al., 2020 c). For instance, $56.0 \%$ anxiety was found, with these participants reporting more than $4 \mathrm{~h}$ of social media exposure, whereas it was $42.1 \%$ for $\leqslant 2 \mathrm{~h}$ (Hossain et al., 2020c). Similarly, a higher depression prevalence rate was observed in those physicians who reported it too hard to stay away from social media $(58.3 \%$ v. 47.8\%) (Hasan et al., 2020).

\section{Physical exercise}

The physical exercise patterns had been changed after the inception of the COVID-19 pandemic, which was reported significantly associated with psychological burdens. For instance, participants who were not involved or inadequately involved with physical exercise generally experienced higher levels of depression and anxiety (Islam et al., 2020b; Khan et al., 2020; Tasnim et al., 2020).

\section{Physical health conditions}

Comorbidities were found to be a significant risk factor during the COVID-19 pandemic, leading to higher mental health problems. In contrast, the presence of several chronic diseases (i.e. hypertension, asthma, diabetes, heart diseases, chronic kidney disease, thyroid disorder) also significantly influenced mental health issues (Barua et al., 2020; Mamun et al., 2020; Rahman et al., 2020; Sakib et al., 2021). Besides, due to more physical health problems, more severe psychological disorders were observed; for instance, it was reported among general people (Mamun et al., 2020).

\section{Psychological problems}

A few studies reported other psychological problems associated with depression, anxiety, and stress. For instance, insomnia has been reported to have influenced the risk of mental health sufferings among university students and general people (Islam et al., 2020b; Mamun et al., 2020). However, the physicians who reported sleeping $<6 \mathrm{~h}$ are significantly associated with higher depression and anxiety symptoms (Hasan et al., 2020).

\section{COVID-19-related risk factors}

\section{Insufficient protective resources}

The shortage of protective tools was a risk factor for psychological distresses during the COVID-19 pandemic. However, few studies reported that the lack of personal protection equipment, inadequate or insufficient training, and other incentives related to protection influenced the risk of mental health problems among healthcare professionals (Barua et al., 2020; Hasan et al., 2020; Tasnim et al., 2020). Besides, Sakib et al. (2021) found that only $15.9 \%$ of the healthcare professionals were satisfied with the quality of the PPE provided by the authorities, and the prevalence of depression was higher among HCPs who were dissatisfied with PPE (30.2\%v. $18.0 \%$ ). However, no studies that reported PPE-related issues in respect to mental health were conducted in other cohorts.

\section{COVID-19-related perceptions}

Peoples' perception of the COVID-19 pandemic significantly impacted their mental health. A study found that participants who had the lowest confidence in their current living place due to the COVID-19 pandemic and were highly concerned about their earning showed enormously significant associations with higher mental health sufferings (Rahman et al., 2020). Whereas, in the case of perception of the current social life, satisfied respondents were less likely to have depression, anxiety, and stress (Rahman et al., 2020). In addition, perceptions related to (i) normal life disruption caused by the COVID-19 pandemic, (ii) its negative mental health effect of the pandemic, (iii) its effects on the healthcare system, leading people not be able to get enough medical care, (iv) for Bangladesh, the COVID-19 pandemic does/will not be a major problem, (v) its negative impact on education, etc., had been observed being associated with mental health problems (Islam et al., 2020c; Sayeed et al., 2020).

\section{Fear of COVID-19 infection}

Most of the studies reported fear of COVID-19 infection influencing the risk of mental health problems, whereas fear of getting infected by the SARS-CoV-2 either by themselves or their family members or relatives were the risk factors (Al Zubayer et al., 2020; Khan et al., 2020; Mamun et al., 2020; Yeasmin et al., 2020; Faisal et al., 2021; Safa et al., 2021; Yasmin et al., 2021). For instance, bankers reported that they were fearful of getting infected by the SARS-CoV-2 as their colleagues became infected, which later developed mental health problems (more specifically depression) among the other bankers (Yasmin et al., 2021).

\section{COVID-19-related symptoms}

People feeling or suffering from the COVID-19-related symptoms were at an increased risk of mental health-related outcomes (Rahman et al., 2020; Sayeed et al., 2020). A study among the students found having more than one symptom had higher odds of stress and anxiety levels compared to those with one symptom (3.06 v. 1.60, and 4.96 v. 3.02, respectively; reference: no symptom) (Sayeed et al., 2020). Correspondingly, another study observed that participants who had COVID-19-like symptoms, including fever, fatigue, and dry cough in the last 14 days, were significantly prone to suffering from depression, anxiety, and stress (Rahman et al., 2020).

\section{Being suspected/infected with the COVID-19}

Individuals who suspected or confirmed that they had close or indirect contact with the confirmed cases of COVID-19 were significantly suffered from higher levels of anxiety, depression, and stress (Rahman et al., 2020). Similarly, 3.17 and 3.99 times higher risk of anxiety and stress suffering were observed in these participants reporting contact with individuals with confirmed cases of COVID-19 (Sayeed et al., 2020). 


\section{Being quarantined or isolated}

Quarantine and isolation are the situations that generally increase the higher risk of mental health problems through developing trauma among the quarantined or isolated individuals. For example, a study found that participants reported being quarantined 3.67-fold anxious than those who were not quarantined (Sayeed et al., 2020). Another study by Ripon et al. (2020) reported that females generally developed a 4.7 and 4.8 times higher risk of PTSD and depression among home-quarantined people than male-quarantined people.

\section{Healthcare facility and patient-related information}

Physicians reporting inadequate resources to deal with the COVID-19-infected patients were at an increased risk of depression and/or anxiety; that is, 2.28, 5.30, and 2.99 times higher risk was predicted for the mild, moderate, and severe levels of depression and/or anxiety, respectively (Barua et al., 2020). Thus, healthcare professionals were being reported restlessness while examining the patients with flu-like symptoms, whereas a double depression rate was identified to these physicians compared to those who were not restless (Sakib et al., 2021). Consequently, physicians who reported to be ready to deal with a COVID-19-positive patient had experienced higher levels of depression and anxiety (Hasan et al., 2020).

\section{Publication bias}

Publication bias was visualized using Funnel plots (Fig. 5), where Egger's test was assessed for detecting publication bias. No significant publication bias was found in terms of depression $(p=0.14)$, anxiety $(p=0.08)$, and stress $(p=0.10)$.

\section{Discussion}

This study, the first systematic review and meta-analysis on common mental health problems during the COVID-19 pandemic in Bangladesh has potential implications for mental health policymaking and practice. It is anticipated that the findings presented herein can be implemented for (i) developing cost-effective mental health strategies, (ii) prioritizing and integrating mental health in existing healthcare systems, (iii) improving access to information and mental health resources, (iv) addressing mental health disparities across different cohorts, (v) mobilizing social and community resources and organizations, (vi) strengthening mental health systems for the forthcoming public health emergencies, etc. (Hossain et al., 2020b).

After investigating a total of 24 recruited studies related to the COVID-19 pandemic, the pooled prevalence of depression, anxiety, and stress was $47 \%$ (95\% CI $\left.39-55 \%, I^{2}=99.14 \%\right)$, $47 \%\left(95 \%\right.$ CI $\left.39-54 \%, I^{2}=99.78 \%\right)$, and $44 \%$ (95\% CI $30-58 \%$, $I^{2}=99.36 \%$ ), respectively. Higher rates of depression were reported among the university student's cohort, that is, $82.4 \%$, and lower rates were reported for bankers (i.e. 12.1\%). Similarly, higher rates of anxiety (81.8\%) were observed among university students, whereas the rate was lower for bankers (10.6\%). In addition, general people reported a higher level of stress (85.6\%), whereas lower was reported among the bankers (11.1\%). Most importantly, the prevalence rates of depression, anxiety, and stress vary within a specific group (general population, for example) due to the utilization of different scales along with discrete cutoff values. Besides this, the sampling method and non-representativeness of the included studies may also limit its reproducibility (al Mamun et al., 2021). In addition, there may be an effect of the participants' area's COVID-19 exposure rate, personal and familiar history of the COVID-19 infection, financial conditions, and so forth lockdown-related issues, which are not possibly identified from the included studies for concluding an overall assumption on mental health problems prevalence rates across the cohorts (al Mamun et al., 2021). However, despite these limitations of the included studies, this review, for the first time, estimated the mental health problems' prevalence rates, which are anticipated to be worthy of consideration for defining the pandemic as a cautious situation rather than factual findings.

The COVID-19 pandemic has dramatically interchanged the consistent activities of individuals to prevent and control the outbreak. Under this circumstance, existing healthcare facilities in the country transformed into increased demands beyond its existence as competent. During the normal period, effective and efficient health care services were allegedly absent in Bangladesh (Anwar et al., 2020; Al-Zaman, 2020). Along with this, due to the shortage of adequate preventive equipment, frontline healthcare professionals frequently feel unsafe and get scared to be infected as they have to directly take care of the COVID-19-infected patients (Hasan et al., 2020). Besides, many frontline healthcare professionals lost their lives worldwide being infected with the SARS-CoV-2 in their workplaces (Bandyopadhyay et al., 2020). Later on, this situation also upsurges psychological weight, including suicidal occurrences among the healthcare providers (Jahan et al., 2021). Consistent with the scenario, the physicians who provided services in the Dhaka division were more susceptible to being anxious than other divisional physicians, which may be due to being identified in most cases and deaths in the Dhaka division.

Applying some of the mandatory restrictions, including social distancing at the earlier period of the pandemic inception, people started grabbing and worrying about their lives and personal safety, fear of getting infected, lost a beloved one, absence of appropriate treatment, etc., which influenced the risk of depression, anxiety, and stress (Hossain et al., 2020a, 2020b). Thus, participants belonging to general people and students cohorts had to experience mental health problems. Besides this, daily wage-earners were at higher risk of adverse psychological effects as they were unable to provide for their families' needs due to the nationwide lockdown and the loss of their jobs (Sultana et al., 2020), where economic crisis and unemployment is reported as the main suicide stressors during the COVID-19 pandemic (Mamun, 2021). However, despite the wage-earners being a risky group, only one study was conducted within this cohort. Similarly, a study was conducted for other vulnerable cohorts, e.g. COVID-19-infected people, COVID-19-suspected people, and bankers. Through the RT-PCR test, the individuals diagnosed with the COVID-19 generally got scared about the mortality risk, which affects their adverse psychological consequences and leads to suicidal situations [please refer to Mamun (2021) for details]. For similar reasons, the quarantined people were found experiencing detraction of psychological well-being (Ripon et al., 2020). In addition, bankers were also at higher risk of being affected psychologically as they had to deal with the clients during the pandemic since the banks were not completely shut down. Therefore, their colleague's news of being infected got scary, although lower prevalence rates of anxiety, depression, and stress were reported in this cohort. Nevertheless of the situation, only one study was directed upon this mentally susceptible group, which may result in vague pictures concluding bankers compared 
to other cohorts are not at risk of higher mental health problems as reported in the only study (Yasmin et al., 2021).

This review also revealed the associated risk factors that influenced the incidence of psychological consequences during the COVID-19 pandemic situation in Bangladesh. Based on the present review, socio-demographic factors including gender (mostly being female), younger people, living in an urban area, family size (more than five members), being unemployed, marital and educational status, having children and elderly in a family influenced the risk of being mentally affected. Furthermore, it was observed that people who had existing physical health-related comorbidities developed higher psychological problems (Barua et al., 2020; Mamun et al., 2020). Besides this, being physically inactive and constantly using social media and watching news reported an increase in the risk of mental instabilities (Hasan et al., 2020; Hossain et al., 2020c). In addition, the COVID19-related perceptions, experiencing the COVID-19-related symptoms, being suspected or infected with the SARS-CoV-2, being contacted with the COVID-19 confirmed cases, being quarantined or isolated, etc., were reported as the psychological suffering risk factors. Finally, the fear of being infected with the virus due to the scarcity of appropriate preventive equipment and inadequate resources in healthcare settings to deal with the COVID-19 infected patients were also reported as a risk factor of those psychological consequences (Hasan et al., 2020). In the end, it can be said that any of the COVID-19-related adverse outcomes increased the risk of higher mental health problems.

Several limitations of this review are yet to be mentioned. First of all, potential studies that could have met this review inclusion criterion were not included beyond the strategies, which may limit the review by selection bias; although databases such as Google Scholar and ResearchGate were used for screening additional articles, and the research team tried their best to sort out all of the eligible articles. Preprint articles were included in this review in view of disseminating the available relevant information; however, these articles being non-peer-reviewed can be one of the limitations of study inclusion. In addition, the study had significant heterogeneity in terms of reporting a pooled prevalence of depression, anxiety, and stress. Using different assessment scales and cutoff values simply explained the heterogeneity found in the review. Additionally, the risk factors associated with mental health problems were estimated from different analyses in the included studies. Despite these limitations, the present review provides an overall estimation of the mental health problems (e.g. depression, anxiety, stress) during the COVID-19 in the context of Bangladesh, which may have potential influences on adopting preventive mental health strategies, including knowledge exchange and training people through employing online-based intervention programs along with providing psychological cares, implementing cohort-based intervention program, arranging series webinar with a special focus on mental health, telecasting positivity about COVID-19 on media channel by taking as caution rather than factual findings.

\section{Concluding remarks}

The COVID-19 pandemic has become a global public health threat due to its devastating effects on peoples' lives worldwide. As an infectious life-threatening pneumonic disease, the pandemic impacted individuals' psychological well-being and physiological departures. This pandemic situation adversely altered the normal life patterns of all sections of people due to its adverse effect and implemented some restrictive measures associated with developing psychological problems such as depression, anxiety, and stress. To facilitate mental health policymaking and practice, there is no beyond integrated information. Therefore, for the first time, the present study depicts the prevalence rates for these mental health outcomes along with its influencing factors, which demand the potential attention of the government of Bangladesh to alleviate these outcomes by implementing some appropriate measures based on specific cohorts; for instance, raising awareness by providing proper knowledge and effective psychological care by a psychologist or psychiatric nurse by organizing onlinebased intervention programs. In addition, the authorities should also telecast positive information on media (such as recovery rate) related to COVID-19 to reduce psychological burdens.

The mental health prevention approach for psychiatric professionals should consist of two major aspects: prevention of mental health issues and mental well-being promotion. The burden of mental health problems can be prevented through three levels (i.e. primary, secondary, or tertiary). Identified risk factors of depression, anxiety, and stress exacerbated by the COVID-19 pandemic situation include socio-economic inequalities, unemployment, poverty, debt, food insecurity, child adversity, parental mental disorder, physical conditions, isolation, and quarantine issues should care at the primary level. Later on, early intervention to reduce the adverse effects and rehabilitation of the suffering people should be considered by education, reducing stigma, etc. Mental health promotional programs (e.g. tele-mental health services, online campaigns, etc.) are suggested considering by psychiatric professionals. However, from this review, the provided baseline information related to prevalence and risk factors of depression, anxiety, and stress across different cohorts can be utilized in the policy levels to adopt appropriate preventive measures and practices to alleviate psychological consequences.

Supplementary material. The supplementary material for this article can be found at https://doi.org/10.1017/gmh.2021.49

Data. All relevant data are within the manuscript cited in the respective places.

Acknowledgements. The authors would like to acknowledge that their affiliation, CHINTA Research Bangladesh, was formerly known as Undergraduate Research Organization.

Financial support. None.

Conflict of interest. None.

Ethical standards. Not applicable.

\section{References}

Ahmed O, Ahmed MZ, Alim SMAHM, Khan MDAU and Jobe MC (2020) COVID-19 outbreak in Bangladesh and associated psychological problems: an online survey. Death Studies, epub ahead of print, 1-7. doi: https://doi. org/10.1080/07481187.2020.1818884

Al-Zaman M (2020) Healthcare crisis in Bangladesh during the COVID-19 pandemic. American Journal of Tropical Medicine and Hygiene 103, 1357-1359. https://doi: 10.4269/ajtmh.20-0826

Al Banna MH, Sayeed A, Kundu S, Christopher E, Hasan MT, Begum MR, Dola STI, Hassan MM, Chowdhury S and Khan MSI (2020) The impact of the COVID-19 pandemic on the mental health of the adult population in Bangladesh: a nationwide cross-sectional study. International Journal of Environmental Research and Public Health 2, 1-12. doi: https://doi.org/ 10.1080/09603123.2020.1802409

al Mamun F, Hosen I, Misti JM, Kaggwa MM and Mamun MA (2021) Mental disorders of Bangladeshi students during the COVID-19 pandemic: a 
systematic review. Psychology Research and Behavior Management 14, 645654. https://doi.org/10.2147/PRBM.S315961

Al Zubayer A, Rahman ME, Islam MB, Babu SZD, Rahman QM, Bhuiyan MRAM, Khan MKA, Chowdhury MAU, Hossain L and Habib RB (2020) Psychological states of Bangladeshi people four months after the COVID-19 pandemic: an online survey. Heliyon 6, e05057. https://doi.org/10.1016/j. heliyon.2020.e05057

Anwar S, Nasrullah M and Hosen MJ (2020) COVID-19 and Bangladesh: challenges and how to address them. Frontiers in Public Health 8, 154 https://doi.org/10.3389/fpubh.2020.00154

Bandyopadhyay S, Baticulon RE, Kadhum M, Alser M, Ojuka DK, Badereddin Y, Kamath A, Parepalli SA, Brown G and Iharchane S (2020) Infection and mortality of healthcare workers worldwide from COVID-19: a systematic review. BMJ Global Health 5, e003097. https:// doi.org/10.1136/bmjgh-2020-003097

Barua L, Zaman MS, Omi FR and Faruque M (2020) Psychological burden of the COVID-19 pandemic and its associated factors among frontline doctors of Bangladesh: a cross-sectional study. F1000Research 9, 1304. https:// doi.org/10.12688/f1000research.27189.3

BBC News (2020) Coronavirus confirmed as pandemic by World Health Organization. https://www.bbc.com/news/world-51839944. Accessed 23 March 2021.

Bhuiyan AKMI, Sakib N, Pakpour AH, Griffiths MD and Mamun MA (2020) COVID-19-related suicides in Bangladesh due to lockdown and economic factors: case study evidence from media reports. International Journal of Mental Health and Addiction, epub ahead of print. 1-6. https://doi.org/10.1007/s11469-020-00307-y

CDC (2020) Symptoms of COVID-19. Available at: https://www.cdc.gov/coronavirus/ 2019-ncov/symptoms-testing/symptoms.html. Accessed 23 March 2021.

Chen N, Zhou M, Dong X, Qu J, Gong F, Han Y, Qiu Y, Wang J, Liu Y and Wei Y (2020) Epidemiological and clinical characteristics of 99 cases of 2019 novel coronavirus pneumonia in Wuhan, China: a descriptive study. Lancet 395, 507-513. https://doi.org/10.1016/S0140-6736(20)30211-7

Dhar BK, Ayittey FK and Sarkar SM (2020) Impact of COVID-19 on psychology among the university students. Global Challenges 4, 2000038. https:// doi.org/10.1002/gch2.202000038

Dsouza DD, Quadros S, Hyderabadwala ZJ and Mamun MA (2020). Aggregated COVID-19 suicide incidences in India: fear of COVID-19 infection is the prominent causative factor. Psychiatry Research 290, 113145 https://doi.org/10.1016/j.psychres.2020.113145

Faisal RA, Jobe MC, Ahmed O and Sharker T (2021) Mental health status, anxiety, and depression levels of Bangladeshi university students during the COVID-19 pandemic. International Journal of Mental Health and Addiction, epub ahead of print. 1-16. https://doi.org/10.1007/s11469-020-00458-y

Gualano MR, Lo Moro G, Voglino G, Bert F and Siliquini R (2020) Effects of Covid-19 lockdown on mental health and sleep disturbances in Italy. International Journal of Environmental Research and Public Health 17, 4779. https://doi.org/10.3390/ijerph17134779

Hasan MT, Hossain S, Safa F, Anjum A, Khan AH, Koly KN, Alam SF, Rafi MA, Podder V and Trisa TI (2020) Prevalence of anxiety and depressive symptoms among physicians during the COVID-19 pandemic in Bangladesh: a crosssectional study. medRxiv. https://doi.org/10.1101/2020.12.08.20245829

Hawryluck L, Gold WL, Robinson S, Pogorski S, Galea S and Styra R (2004) SARS control and psychological effects of quarantine, Toronto, Canada. Emerging Infectious Diseases 10, 1206. https://doi.org/10.3201/eid1007. 030703

Hossain MM, Rahman M, Trisha NF, Tasnim S, Nuzhath T, Hasan NT, Clark H, Das A, McKyer ELJ and Ahmed HU (2020a) Prevalence of anxiety and depression in South Asia during COVID-19: a systematic review and meta-analysis. PsyArXiv Prepr. https://doi.org/10.31234/osf.io/q4k5b

Hossain MM, Tasnim S, Sultana A, Faizah F, Mazumder H, Zou L, McKyer ELJ, Ahmed HU and Ma P (2020b) Epidemiology of mental health problems in COVID-19: a review. F1000Research 9, 636. https://doi.org/10. 12688/f1000research.24457.1

Hossain MT, Ahammed B, Chanda SK, Jahan N, Ela MZ and Islam MN $(2020 c)$ Social and electronic media exposure and generalized anxiety disorder among people during COVID-19 outbreak in Bangladesh: a preliminary observation. PLoS ONE 15, e0238974. https://doi.org/10.1371/ journal.pone.0238974

Islam MS, Ferdous MZ and Potenza MN (2020a) Panic and generalized anxiety during the COVID-19 pandemic among Bangladeshi people: an online pilot survey early in the outbreak. Journal of Affective Disorders 276, 30-37. https://doi.org/10.1016/j.jad.2020.06.049

Islam MS, Sujan MSH, Tasnim R, Sikder MT, Potenza MN and van Os J (2020b) Psychological responses during the COVID-19 outbreak among university students in Bangladesh. PLOS ONE 15, e0245083. https://doi. org/10.1371/journal.pone.0245083

Islam SMD-U, Bodrud-Doza M, Khan RM, Haque MA and Mamun MA (2020c) Exploring COVID-19 stress and its factors in Bangladesh: a perception-based study. Heliyon 6, e04399. https://doi.org/10.1016/j.heliyon.2020.e04399

Islam MA, Barna SD, Raihan H, Khan MNA and Hossain MT (2020d) Depression and anxiety among university students during the COVID-19 pandemic in Bangladesh: a web-based cross-sectional survey. PLoS ONE 15, e0238162. https://doi.org/10.1371/journal.pone.0238162

Jahan I, Ullah I, Griffiths MD and Mamun MA (2021) COVID-19 suicide and its causative factors among the healthcare professionals: case study evidence from press reports. Perspectives in Psychiatric Care, 57, 1707-1711. https://doi.org/10.1111/ppc.12739

Khan AH, Sultana MS, Hossain S, Hasan MT, Ahmed HU and Sikder MT (2020) The impact of COVID-19 pandemic on mental health \& well-being among home-quarantined Bangladeshi students: a cross-sectional pilot study. Journal of Affective Disorders 277, 121-128. https://doi.org/10. 1016/j.jad.2020.07.135

Khatun MF, Parvin MF, Rashid MM, Alam MS, Talukder A, Razu SR, Ward PR and Ali M (2021) Mental health of physicians during COVID-19 outbreak in Bangladesh: a web-based cross-sectional survey. Frontiers in Public Health 9, 592058. doi: 10.3389/fpubh.2021.592058

Mamun MA (2021) Suicide and suicidal behaviors in the context of COVID-19 pandemic in Bangladesh: a systematic review. Psychology Research and Behavior Management 14, 695-704. https://doi.org/10.2147/ PRBM.S315760

Mamun MA and Griffiths MD (2020) First COVID-19 suicide case in Bangladesh due to fear of COVID-19 and xenophobia: possible suicide prevention strategies. Asian Journal of Psychiatry 51, 102073. https://doi.org/ 10.1016/j.ajp.2020.102073

Mamun MA, Sakib N, Gozal D, Bhuiyan AKMI, Hossain S, Bodrud-Doza M, Al Mamun F, Hosen I, Safiq MB and Abdullah AH (2020) The COVID-19 pandemic and serious psychological consequences in Bangladesh: a population-based nationwide study. Journal of Affective Disorders 279, 462-472. https://doi.org/10.1016/j.jad.2020.10.036

Mina FB, Billah M, Karmakar S, Das S, Rahman MS, Hasan MF and Acharjee UK (2021) An online observational study assessing clinical characteristics and impacts of the COVID-19 pandemic on mental health: a perspective study from Bangladesh. Journal of Public Health, epub ahead of print. https://doi.org/10.1007/s10389-020-01445-2

Moher D, Liberati A, Tetzlaff J and Altman DG (2010) Preferred reporting items for systematic reviews and meta-analyses: the PRISMA statement. International Journal of Surgery 8, 336-341. https://doi.org/10.1016/j.ijsu. 2010.02.007

Puspitasari IM, Yusuf L, Sinuraya RK, Abdulah R and Koyama H (2020) Knowledge, attitude, and practice during the COVID-19 pandemic: a review. Journal of Multidisciplinary Healthcare 13, 727-733. https://doi. org/10.2147/JMDH.S265527

Rahman MM, Khan SJ, Sakib MS, Chakma S, Procheta NF, Mamun ZA, Arony A, Rahman F and Rahman MM (2020) Assessing the psychological condition among general people of Bangladesh during COVID-19 pandemic. Journal of Human Behavior in the Social Environment, epub ahead of print. https://doi.org/10.1080/10911359.2020.1848688

Ripon RK, El-Sabban F, Sikder T, Hossain S, Mim SS, Ahmed HU and Mehta N (2020) Psychological and nutritional effects on a COVID-19-quarantined population in Bangladesh. Journal of Human Behavior in the Social Environment 31, 271-282. https://doi.org/10.1080/ 10911359.2020.1822252 
Safa F, Anjum A, Hossain S, Trisa TI, Alam SF, Abdur Rafi M, Podder V, Koly KN, Azad DT, Ahmad WU, Nodi RN, Ashraf F, Quamrul Akhter SM, Ahmed HU and Hasan MT (2021) Immediate psychological responses during the initial period of the COVID-19 pandemic among Bangladeshi medical students. Children and Youth Services Review 122, 105912. https://doi.org/10.1016/j.childyouth.2020.105912

Sakib N, Akter T, Zohra F, Bhuiyan AKMI, Mamun MA and Griffiths MD (2021) Fear of COVID-19 and depression: a comparative study among the general population and healthcare professionals during COVID-19 pandemic crisis in Bangladesh. International Journal of Mental Health and Addiction, epub ahead of print. https://doi.org/10.1007/s11469020-00477-9

Sayeed A, Kundu S, Banna MHA, Hasan MT, Begum MR and Khan MSI (2020) Mental health outcomes during the COVID-19 and perceptions towards the pandemic: findings from a cross sectional study among Bangladeshi students. Children and Youth Services Review 119, 105658. https://doi.org/10.1016/j.childyouth.2020.105658

Sultana MS, Khan AH, Hossain S, Islam T, Hasan MT, Ahmed HU and Khan JAM (2020) The association between financial hardship and mental health difficulties among adult wage earners during the COVID-19 pandemic in Bangladesh: findings from a cross-sectional analysis. PsyArXiv. https://doi.org/10.31234/osf.io/q3ehv
Sundarasen S, Chinna K, Kamaludin K, Nurunnabi M, Baloch GM, Khoshaim HB, Hossain SFA and Sukayt A (2020) Psychological impact of Covid-19 and lockdown among university students in Malaysia: implications and policy recommendations. International Journal of Environmental Research and Public Health 17, 6206. https://doi.org/10.3390/ijerph 17176206

Tasnim R, Sujan MSH, Islam MS, Ritu AH, Siddique MAB, Toma TY, Nowshin R, Hasan A, Hossain S and Nahar S (2020) Prevalence and correlates of anxiety and depression in frontline healthcare workers treating people with COVID-19 in Bangladesh. PsyArXiv. https://doi.org/10.31234/osf.io/3qg9p

World Health Organization (2020) COVID-19 disrupting mental health services in most countries, WHO survey. Available at: https://www.who.int/ news/item/05-10-2020-covid-19-disrupting-mental-health-services-in-mostcountries-who-survey. Accessed 23 March 21.

Yasmin S, Alam MK, Ali FB, Banik R and Salma N (2021) Psychological impact of COVID-19 among people from the banking sector in Bangladesh: a cross-sectional study. International Journal of Mental Health and Addiction, epub ahead of print. https://doi.org/10.1007/s11469-020-00456-0

Yeasmin S, Banik R, Hossain S, Hossain MN, Mahumud R, Salma N and Hossain MM (2020) Impact of COVID-19 pandemic on the mental health of children in Bangladesh: a cross-sectional study. Children and Youth Services Review 117, 105277. https://doi.org/10.1016/j.childyouth.2020.105277 